Fortschritt-Berichte VDl

VDI

Reihe 12

Verkehrstechnik/ Fahrzeugtechnik

Nr. 809
M.Sc. Jakob Philipp Bechtloff, Ludwigsburg
Schätzung des

Schwimmwinkels und

fahrdynamischer

Parameter zur

Verbesserung

modellbasierter

Fahrdynamikregelungen

Berichte aus dem

Institut für

Automatisierungstechnik und Mechatronik der TU Darmstadt

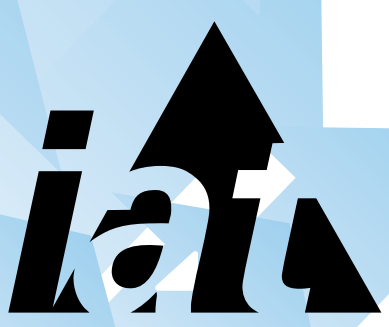


https://doi.org/10.51202/9783186809124-1

Generiert durch IP '172.22.53.54', am 26.04.2023, 11:44:44.

Das Erstellen und Weitergeben von Kopien dieses PDFs ist nicht zulässig. 


\title{
Schätzung des Schwimmwinkels und fahrdynamischer Parameter zur Verbesserung modellbasierter Fahrdynamikregelungen
}

\author{
Dem Fachbereich \\ Elektrotechnik und Informationstechnik \\ der Technischen Universität Darmstadt \\ zur Erlangung des Grades eines Doktor-Ingenieurs (Dr.-Ing.) \\ vorgelegte Dissertation \\ von \\ Jakob Philipp Bechtloff, M.Sc. \\ geboren am 12. März 1988 in Peine
}

Referent: $\quad$ Prof. Dr.-Ing. Dr. h. c. Rolf Isermann

Korreferent: $\quad$ Prof. Dr.-Ing. Jürgen Adamy

Tag der Einreichung: 19. September 2017

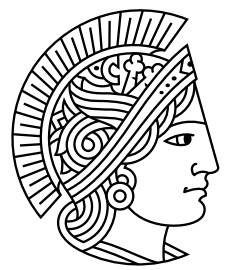

D $17 \cdot$ Darmstadt 
https://doi.org/10.51202/9783186809124-1

Generiert durch IP '172.22.53.54', am 26.04.2023, 11:44:44.

Das Erstellen und Weitergeben von Kopien dieses PDFs ist nicht zulässig. 


\section{Fortschritt-Berichte VDI}

\section{Reihe 12}

Verkehrstechnik/

Fahrzeugtechnik
M.Sc. Jakob Philipp Bechtloff, Ludwigsburg

Nr. 809

Schätzung des

Schwimmwinkels und fahrdynamischer

Parameter zur

Verbesserung

modellbasierter

Fahrdynamikregelungen

Berichte aus dem

Institut für

Automatisierungstechnik und Mechatronik der TU Darmstadt

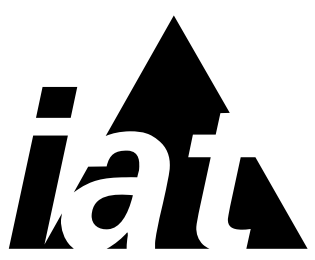


Bechtloff, Jakob Philipp

\section{Schätzung des Schwimmwinkels und fahrdynamischer Parameter zur Verbesserung modellbasierter Fahrdynamikregelungen}

Fortschr.-Ber. VDI Reihe 12 Nr. 809. Düsseldorf: VDI Verlag 2018. 176 Seiten, 93 Bilder, 1 Tabelle.

ISBN 978-3-18-380912-7, ISSN 0178-9449, $€ 62,00 / \mathrm{VDI}-$ Mitgliederpreis $€ 55,80$.

Für die Dokumentation: Nichtlineare Zustandsschätzung - Fahrzustandsschätzung - Fahrdynamikbeobachter - Unscented Kalman-Filter - Fahrdynamikmodelle - Sensorfusion - Schwimmwinkelschätzung - Reibwertschätzung - Schräglaufsteifigkeiten - Fahrdynamikregelsysteme

Die vorliegende Arbeit wendet sich an Ingenieure und Wissenschaftler im Bereich der Fahrdynamikregelungen. Sie befasst sich mit der Entwicklung einer Methode zur Schätzung der nichtmessbaren Bewegungsgrößen Schwerpunktgeschwindigkeit und Schwimmwinkel. Dafür wird lediglich die Sensorik der Electronic Stability Control genutzt. Damit eine modellbasierte Steuerung oder ein Führungsmodell auf Veränderung des querdynamischen Fahrverhaltens reagieren kann, werden zusätzlich die fahrdynamisch wichtigsten Parameter, wie die Schräglaufsteifigkeiten und der maximale Reibwert während des Fahrbetriebs geschätzt. Hierzu werden Fahrdynamikmodelle entwickelt, die alle erforderlichen Situationen, wie die Fahrt in der Steilkurve, Fahrten im physikalischen Grenzbereich auf Hoch- und Niedrigreibwert genau genug modellieren. Es wird gezeigt, warum ein Unscented Kalman-Filter im fahrdynamischen Grenzbereich durch die Berücksichtigung der Nichtlinearität der Achsquerkraftkennlinien den Schwimmwinkel robuster als der erweiterte Kalman-Filter schätzt.

\section{Bibliographische Information der Deutschen Bibliothek}

Die Deutsche Bibliothek verzeichnet diese Publikation in der Deutschen Nationalbibliographie; detaillierte bibliographische Daten sind im Internet unter www.dnb.de abrufbar.

\section{Bibliographic information published by the Deutsche Bibliothek}

(German National Library)

The Deutsche Bibliothek lists this publication in the Deutsche Nationalbibliographie

(German National Bibliography); detailed bibliographic data is available via Internet at www.dnb.de.

Alle Rechte, auch das des auszugsweisen Nachdruckes, der auszugsweisen oder vollständigen Wiedergabe (Fotokopie, Mikrokopie), der Speicherung in Datenverarbeitungsanlagen, im Internet und das der Übersetzung, vorbehalten.

Als Manuskript gedruckt. Printed in Germany.

ISSN 0178-9449

ISBN 978-3-18-380912-7 


\section{Vorwort}

Diese Arbeit entstand im Rahmen meiner Tätigkeit als wissenschaftlicher Mitarbeiter bei Prof. Dr.-Ing. Dr. h.c. Isermann am Institut für Regelungstechnik und Mechatronik der TU Darmstadt im Rahmen einer Forschungskooperation mit der Bosch Engineering GmbH.

Mein Dank gilt zuallererst Herrn Prof. Isermann für die wissenschaftliche Betreuung sowie den Freiraum, den er mir bei der Bearbeitung dieser Aufgabe ließ. Durch seine angenehme und gelassene Art ist er für mich auch menschlich ein Vorbild geworden.

Ich danke Herrn Prof. Adamy für die Übernahme des Korreferats und das Interesse an meiner Arbeit.

Danken möchte ich auch den Mitarbeitern der Bosch Engineering GmbH, die in vielen Gesprächen und Diskussionen anregende Impulse gaben und so wesentlich zum Gelingen dieser Arbeit beitrugen. Vor allem möchte ich mich bei Dr. König bedanken. Er war der Initiator für die Forschungskooperation und konnte mich immer wieder motivieren und mit seinem fahrdynamischen Wissen unterstützen.

Ich möchte mich auch bei all meinen Kollegen am Institut für Automatisierungstechnik für das familiäre Arbeitsklima bedanken. Mit den Jahren sind echte Freundschaften entstanden. Für ihre Arbeit im Rahmen von Seminar- und Abschlussarbeiten möchte ich mich bei meinen ehemaligen Studenten bedanken. Herausheben möchte ich hier Sven Pospischil, dessen Arbeit ein Teil meiner Dissertation wurde. Ebenfalls möchte ich mich beim Team vom Sekretariat Ilse Brauer, Corinna Fischer und ganz besonders bei Brigitte Hoppe bedanken, die die Arbeit Korrektur gelesen hat.

Ein riesiger Dank gilt meinen Eltern, die es mir ermöglichten Maschinenbau und Mechatronik zu studieren. Allerdings schufen sie schon viel früher die Basis für diese Arbeit, indem sie mir große Freiräume ließen, meine Leidenschaft für die Geschwindigkeit und das Gefühl für den fahrdynamischen Grenzbereich von Kind an auszuleben. Mein Vater ließ sich im Laufe der Promotion auch von den Kalman-Filtern begeistern, sodass wir immer wieder anregende fachliche Diskussionen führen konnten. Meine Mutter gab mir den nötigen Halt und ermutigte mich, wenn meine Motivation auch mal nachließ.

Ludwigsburg, Januar 2018

Jakob Bechtloff 


\section{Inhaltsverzeichnis}

$\begin{array}{llll}\text { Symbole und Abkürzungen } & \text { VIII }\end{array}$

Kurzfassung

1 Einführung 1

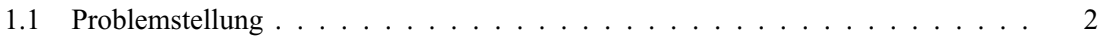

1.2 Zielsetzung und Stand der Technik . . . . . . . . . . . . . . . . . . . 2

1.2 .1 Übergrundgeschwindigkeit . . . . . . . . . . . . . . . . . . . . . 2

1.2 .2 Schwimmwinkel . . . . . . . . . . . . . . . . 4

1.2 .3 Maximaler Reibwert . . . . . . . . . . . . . . . . . . . . . . . . . 11

1.2 .4 Schräglaufsteifigkeiten . . . . . . . . . . . . . . . . . . 14

1.2.5 Achsindividuelle maximale Reibwerte in Querrichtung . . . . . . . . . . 17

1.3 Konzeptbeschreibung und Gliederung der Arbeit . . . . . . . . . . . . . . . 18

2 Modellbildung 21

2.1 Koordinatensysteme . . . . . . . . . . . . . . . . . . . . . . . . . . . . . . . . .

2.2 Lagedarstellung . . . . . . . . . . . . . . . . . . . . . . . . . . . . . . . . . . . . . . 22

2.3 Reifen- und Achskraftmodelle . . . . . . . . . . . . . . . . . . 23

2.3.1 Reifenverhalten . . . . . . . . . . . . . . . . . 24

2.3.2 Effektives Achsquerkraftmodell . . . . . . . . . . . . . . 25

2.3.3 Transientes Querkraftverhalten . . . . . . . . . . . . . . . . . 29

2.3 .4 Reifenlängskraftmodell . . . . . . . . . . . . . . . . . . 30

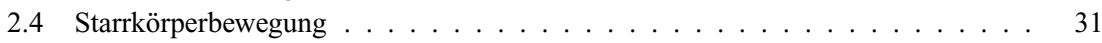

2.4.1 Beispiel: Steilkurve . . . . . . . . . . . . . . . . 33

2.5 Zweispurmodell . . . . . . . . . . . . . . . . . . 34

2.6 Erweitertes Einspurmodell . . . . . . . . . . . . . . . . . . . . 40

2.7 Lineares Einspurmodell . . . . . . . . . . . . . . . . . . . . . . . . 41

2.8 Wankmodell . . . . . . . . . . . . . . . . . . . . . 44

2.9 Zusammenfassung . . . . . . . . . . . . . . . . . 45

3 Grundlagen der Zustandsschätzung $\quad 46$

3.1 Erweitertes Kalman-Filter . . . . . . . . . . . . . . . . . . . . . . 47

3.2 Unscented Kalman-Filter . . . . . . . . . . . . . . . . . . . . . . . . 48

3.3 Vergleich des EKF mit dem UKF . . . . . . . . . . . . . . . . . . . . 50

4 Identifikation $\mathbf{5 3}$

4.1 Grundparameter . . . . . . . . . . . . . . . . . . . . . . . . . . . . . . . 53

4.2 Referenzmesssystem . . . . . . . . . . . . . . . . . 54 


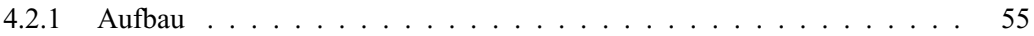

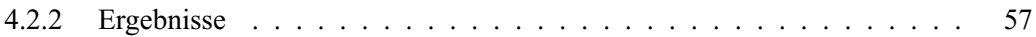

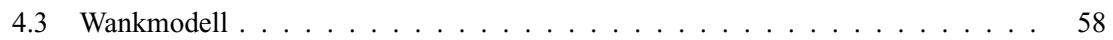

4.4 Achsquerkraftmodelle . . . . . . . . . . . . . . . . . . . . . 59

4.4 .1 Vorgehen . . . . . . . . . . . . . . . . . 59

4.4.2 Identifikation ohne Einfluss von Längsschlupf . . . . . . . . . . . . . . . . . . 63

4.4 .3 Identifikation bei kombiniertem Schlupf . . . . . . . . . . . . . . . 68

4.5 Achslängskraftmodelle . . . . . . . . . . . . . . . . . 71

4.6 Validierung und Vergleich der Einspurfahrdynamikmodelle . . . . . . . . . . 72

4.6 .1 Stationäres Verhalten . . . . . . . . . . . . . . . . . . . . 73

4.6 .2 Bremsen in der Kurve . . . . . . . . . . . . . . . . . . . . . . . . . . . . . . . . . . . . . . . . . 74

4.6 .3 Dynamisches Verhalten . . . . . . . . . . . . . . . . . . . . . 75

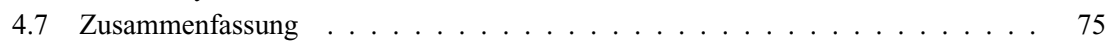

5 Schätzung fahrdynamischer Zustände und Parameter $\quad 77$

5.1 Aufbau der Kalman-Filter . . . . . . . . . . . . . . . . . . . . . . . . . . . . . 77

5.2 Prädiktion . . . . . . . . . . . . . . . . . . . . . . . . . . . . . . . . . . . .

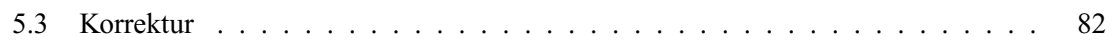

5.3.1 Einspurmodellvorderradgeschwindigkeit . . . . . . . . . . . 83

5.3 .2 Achslängskräfte . . . . . . . . . . . . . . . . . . . . . 84

5.3 .3 Achsquerkräfte . . . . . . . . . . . . . . . . . 86

5.3.4 Schwimmwinkel für niedrige Geschwindigkeiten . . . . . . . . . . . . . 88

5.4 Güte der Schätzung der Geschwindigkeit und der Fahrbahnsteigung . . . . . . . . 91

5.5 Güte der Schätzung des Schwimmwinkels und des Fahrbahnwankwinkels . . . . 95

5.6 Vergleich der Schwimmwinkelschätzung mit EKF und UKF . . . . . . . . . . . 97

5.7 Zusammenfassung . . . . . . . . . . . . . . . . 100

6 Validierungsergebnisse für verschiedene Fahrmanöver 102

6.1 Geschwindigkeitsschätzung . . . . . . . . . . . . . . . . . . . 102

6.2 Schwimmwinkel- und Reibwertschätzung . . . . . . . . . . . . . . . . . . . . . . . . . . . . . . . . . . . .

6.2 .1 Stationäre Kreisfahrt . . . . . . . . . . . . . . . . . . . . . . . . 105

6.2 .2 Doppelspurwechsel . . . . . . . . . . . . . . . . 105

6.2 .3 Steilkurve . . . . . . . . . . . . . . . . . . 106

6.2 .4 Handlingkurs . . . . . . . . . . . . . . . . . . . 110

6.2 .5 Driften . . . . . . . . . . . . . . . . . . . 110

6.2 .6 Niedrigreibwert . . . . . . . . . . . . . . . . . . . . 111

6.2 .7 Zusammenfassung . . . . . . . . . . . . . . . . . 115

6.3 Schätzung der Schräglaufsteifigkeiten . . . . . . . . . . . . . . . . 120

6.3.1 Adaption von zufälligen Startwerten auf Winterbereifung . . . . . . . . . 120

6.3.2 Adaption von Winter- auf Sommerbereifung . . . . . . . . . . . . . . 122

6.3.3 Vergleich mit dem EKF und modularer Parameterschätzung . . . . . . . 123

6.4 Zusammenfassung . . . . . . . . . . . . . . . . 125 
7 Adaptive modellbasierten Fahrdynamikregelung auf Basis der geschätzten Größen

7.1 Adaptive flachheitsbasierte Modellfolgesteuerung . . . . . . . . . . . . . . 127

7.1.1 Entwurf einer Steuerung für flache Systeme . . . . . . . . . . . . . . . 128

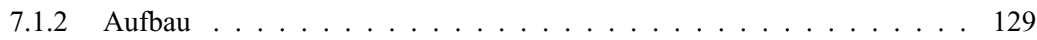

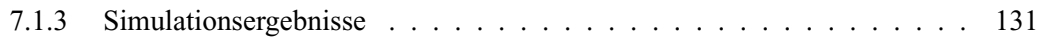

7.2 Schwimmwinkel- und Gierratenregelung durch Bremseingriffe . . . . . . . . . . 134

7.2.1 Aufbau der Gierraten- und Schwimmwinkelregelung . . . . . . . . . . . 135

7.2 .2 Simulationsergebnisse . . . . . . . . . . . . . . 138

7.3 Kombinierte Schwimmwinkel und Gierraten-Steuerung und Regelung . . . . . . 139

7.3.1 Performancesteigerung durch kombinierte Lenk- und Bremseingriffe . . 140

7.3.2 Virtueller Fahrversuch . . . . . . . . . . . . . . . . . 141

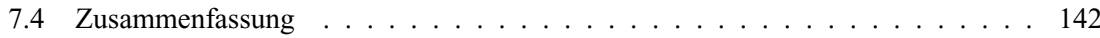

8 Zusammenfassung und Ausblick $\quad 143$

9 Anhang 146

9.1 Definition der Flachheit . . . . . . . . . . . . . . . . . . . . . 146

9.2 Übergang zur Geschwindigkeits-/ Schwimmwinkel-Darstellung des Zweispurmodells . . . . . . . . . . . . . . . . . . . . . 146

9.3 Verwendete Zusatzsensorik . . . . . . . . . . . . . . . . . . . . . 148

$\begin{array}{ll}\text { Literaturverzeichnis } & 151\end{array}$

$\begin{array}{lr}\text { Eigene Veröffentlichungen } & 159\end{array}$

$\begin{array}{lr}\text { Betreute studentische Arbeiten } & 160\end{array}$ 


\section{Symbole und Abkürzungen}

\section{Lateinische Symbole und Formelzeichen}

Symbol Beschreibung

Einheit

\begin{tabular}{|c|c|c|}
\hline $\mathbf{A}$ & Systemmatrix & \\
\hline$A_{\mathrm{X}}$ & Fahrzeugstirnfläche & \\
\hline$a$ & Beschleunigung & $\mathrm{m} / \mathrm{s}^{2}$ \\
\hline$a_{\mathrm{c}}$ & Zentripetalbeschleunigung & $\mathrm{m} / \mathrm{s}^{2}$ \\
\hline B & Eingangsmatrix & \\
\hline$B$ & Pacejka-Reifenmodell-Parameter für die Steigung & \\
\hline$b$ & mittlere Spurweite & $\mathrm{m}$ \\
\hline$b_{\mathrm{f}}$ & Spurweite vorne & $\mathrm{m}$ \\
\hline$b_{\mathrm{r}}$ & Spurweite hinten & $\mathrm{m}$ \\
\hline$b_{\mathrm{S}}$ & Abstand Sensor - Schwerpunkt in Y-Richtung & $\mathrm{m}$ \\
\hline$C$ & Pacejka-Reifenmodell-Formparameter & \\
\hline$C_{\text {Roll }}$ & Parameter für die Wankmomentverteilung & \\
\hline$c_{\mathrm{W}}$ & Luftwiderstandsbeiwert & \\
\hline$c_{\mathrm{SX}_{\mathrm{X}}}$ & Schlupfsteifigkeit & $\mathrm{N}$ \\
\hline$c_{\mathrm{sX}_{\mathrm{X}}}^{*}$ & auf die Radlast bezogene Schlupfsteifigkeit & \\
\hline$c_{\alpha}$ & Schräglaufsteifigkeit & $\mathrm{N} / \mathrm{rad}$ \\
\hline$D$ & Pacejka-Reifenmodell-Parameter für das Kraftmaximum & \\
\hline$D_{\text {Roll }}$ & Dämpfung des Wankmodells & $\mathrm{rad} /(\mathrm{m} / \mathrm{s})$ \\
\hline$E$ & Pacejka-Reifenmodell-Formparameter & \\
\hline$F$ & Kraft & $\mathrm{N}$ \\
\hline$F_{\mathrm{T}}$ & Reifenkraft & $\mathrm{N}$ \\
\hline$F_{\mathrm{R}}$ & Rollwiderstandskraft & $\mathrm{N}$ \\
\hline$g$ & Gravitationskonstante & $\mathrm{m} / \mathrm{s}^{2}$ \\
\hline $\mathbf{H}$ & Ausgangsmatrix & \\
\hline $\mathbf{h}$ & Ausgangsfunktionsvektor & \\
\hline$h$ & Schwerpunkthöhe des Fahrzeugs & $\mathrm{m}$ \\
\hline$h_{\mathrm{S}}$ & Höhe des Sensors & $\mathrm{m}$ \\
\hline$i_{\mathrm{S}}$ & Lenkübersetzung & \\
\hline$J$ & Trägheitsmoment & $\mathrm{kgm}^{2} / \mathrm{rad}$ \\
\hline $\mathbf{K}$ & Kalman-Verstärkung & \\
\hline$K_{\text {Roll }}$ & Verstärkung des Wankmodells & $\mathrm{rad} /\left(\mathrm{m} / \mathrm{s}^{2}\right)$ \\
\hline$k_{\alpha}$ & Quotient aus Querkraft und Schräglaufsteifigkeit & \\
\hline$l$ & Radstand & $\mathrm{m}$ \\
\hline
\end{tabular}




\begin{tabular}{|c|c|c|}
\hline Symbol & Beschreibung & Einheit \\
\hline$l_{\mathrm{r}}$ & Abstand Hinterachse - Fahrzeugschwerpunkt & $\mathrm{m}$ \\
\hline$l_{\mathrm{f}}$ & Abstand Vorderachse - Fahrzeugschwerpunkt & $\mathrm{m}$ \\
\hline$l_{\mathrm{S}}$ & Abstand Sensor - Schwerpunkt in X-Richtung & $\mathrm{m}$ \\
\hline$l_{\mathrm{T}}$ & Einlauflänge & $\mathrm{m}$ \\
\hline$M$ & Drehmoment & $\mathrm{Nm}$ \\
\hline$M_{\mathrm{D}}$ & Antriebsmoment & $\mathrm{Nm}$ \\
\hline$M_{\mathrm{B}}$ & Bremsmoment & $\mathrm{Nm}$ \\
\hline$m$ & Gesamtfahrzeugmasse & $\mathrm{kg}$ \\
\hline $\mathbf{P}$ & Kovarianzmatrix des Schätzfehlers & \\
\hline $\mathbf{P}_{\mathrm{xy}}$ & Kreuzkovarianzmatrix zwischen Schätzfehler und Ausgang & \\
\hline $\mathbf{P}_{\mathrm{zz}}$ & Kovarianzmatrix der Messung & \\
\hline $\mathbf{p}$ & Parametervektor & \\
\hline $\mathbf{Q}$ & Kovarianzmatrix des Prozessrausches & \\
\hline $\mathbf{R}$ & Kovarianzmatrix des Messrausches & \\
\hline$R$ & Kurvenradius & $\mathrm{m}$ \\
\hline$r_{\text {dyn }}$ & dynamischer Reifenhalbmesser & $\mathrm{m}$ \\
\hline$s_{\mathrm{Y}}$ & Querschlupf & \\
\hline$s_{\mathrm{X}}$ & Längsschlupf & \\
\hline$s$ & resultierender Schlupf & \\
\hline$s_{\mathrm{X}, \text { crit }}$ & kritischer Schlupf & \\
\hline $\mathbf{T}$ & Transformationsmatrix & \\
\hline$T_{0}$ & Abtastzeit & $\mathrm{s}$ \\
\hline$T_{\text {Roll }}$ & Zeitkonstante des Wankmodells & $\mathrm{s}$ \\
\hline$t$ & Zeit & $\mathrm{s}$ \\
\hline $\mathbf{u}$ & Eingangsvektor & \\
\hline $\mathbf{v}$ & Messrauschvektor & \\
\hline$v$ & Schwerpunktgeschwindigkeit & $\mathrm{m} / \mathrm{s}$ \\
\hline W & Jacobimatrix des Prozessrauschens & \\
\hline $\mathbf{w}$ & Prozessrauschvektor & \\
\hline $\mathbf{X}$ & Sigma-Partikel & \\
\hline $\mathbf{x}$ & Zustandsvektor & \\
\hline$\hat{\mathbf{x}}$ & geschätzter Zustandsvektor & \\
\hline$x_{\mathrm{E}}$ & laterale Position & $\mathrm{m}$ \\
\hline $\mathbf{Y}$ & Sigma-Partikel durch die Ausgangsfunktion transformiert & \\
\hline $\mathbf{y}$ & Systemausgang & \\
\hline$y_{\mathrm{E}}$ & longitudinale Position & $\mathrm{m}$ \\
\hline $\mathbf{z}$ & Messvektor & \\
\hline
\end{tabular}




\section{Griechische Symbole und Formelzeichen}

\begin{tabular}{|c|c|c|}
\hline Symbol & Beschreibung & Einheit \\
\hline$\alpha$ & Schräglaufwinkel & $\mathrm{rad}$ \\
\hline$\alpha_{\text {crit }}$ & Schräglaufwinkel bei dem die maximale Querkraft entsteht & $\mathrm{rad}$ \\
\hline$\alpha_{\mathrm{f}}$ & Schräglaufwinkel an der Vorderachse & $\mathrm{rad}$ \\
\hline$\alpha_{\mathrm{ij}}$ & Schräglaufwinkel & $\mathrm{rad}$ \\
\hline$\alpha_{\mathrm{r}}$ & Schräglaufwinkel an der Hinterachse & $\mathrm{rad}$ \\
\hline$\beta$ & $\begin{array}{l}\text { Schwimmwinkel in Aufbau-Koordinaten (DIN-ISO-8855:2013-11 } \\
\text { (2013) bezieht sich auf die Ebene) }\end{array}$ & $\mathrm{rad}$ \\
\hline$\dot{\beta}$ & $\begin{array}{l}\text { Schwimmwinkelgeschwindigkeit in Aufbau-Koordinaten (DIN-ISO- } \\
8855: 2013-11 \text { (2013) bezieht sich auf die Ebene) }\end{array}$ & $\mathrm{rad} / \mathrm{s}$ \\
\hline$\delta_{\mathrm{f}}$ & mittlerer Lenkwinkel an der Vorderachse & $\mathrm{rad}$ \\
\hline$\delta_{\mathrm{H}}$ & Lenkradwinkel & $\mathrm{rad}$ \\
\hline$\delta_{\mathrm{r}}$ & mittlerer Lenkwinkel an der Hinterachse & $\mathrm{rad}$ \\
\hline$\theta$ & Parametervektor & \\
\hline$\theta$ & Nickwinkel des Aufbaus relativ zur horizontalen Ebene & $\mathrm{rad}$ \\
\hline$\theta_{\mathrm{K}}$ & Nickwinkel des Aufbaus relativ zur Fahrbahnebene & $\mathrm{rad}$ \\
\hline$\theta_{\mathrm{T}}$ & Nickwinkel der Fahrbahnebene relativ zur horizontalen Ebene & $\mathrm{rad}$ \\
\hline$\kappa$ & $\begin{array}{l}\text { Parameter des Achsquerkraftmodells zur Parametrierung des Längs- } \\
\text { schlupfeinflusses }\end{array}$ & \\
\hline$\mu_{\mathrm{ij}}$ & resultierender Kraftschlussbeiwert am Rad ij, ij $\in\{\mathrm{fl}, \mathrm{fr}, \mathrm{rl}, \mathrm{rr}\}$ & \\
\hline$\mu_{\max , \mathrm{i}}$ & maximaler Kraftschlussbeiwert an Achse $i, i \in\{f, r\}$ & \\
\hline$\xi$ & Regressionsvektor & \\
\hline$\rho$ & Luftdichte & $\mathrm{kg} / \mathrm{m}^{3}$ \\
\hline$\sigma$ & Standartabweichung & \\
\hline$\varphi$ & Wankwinkel des Aufbaus relativ zur horizontalen Ebene & $\mathrm{rad}$ \\
\hline$\varphi_{\mathrm{K}}$ & Wankwinkel des Aufbaus relativ zur Fahrbahnebene & $\mathrm{rad}$ \\
\hline$\varphi_{\mathrm{T}}$ & Wankwinkel der Fahrbahnebene relativ zur horizontalen Ebene & $\mathrm{rad}$ \\
\hline$\Psi$ & Vektor der Eulerwinkel & $\mathrm{rad}$ \\
\hline$\psi$ & Gierwinkel zum erdfesten Koordinatensystem & $\mathrm{rad}$ \\
\hline$\dot{\psi}$ & Gierrate in der Ebene & $\mathrm{rad} / \mathrm{s}$ \\
\hline$\ddot{\psi}$ & Gierbeschleunigung in der Ebene & $\mathrm{rad} / \mathrm{s}^{2}$ \\
\hline$\omega_{\mathrm{ij}}$ & Raddrehgeschwindigkeit & $\mathrm{rad} / \mathrm{s}$ \\
\hline$\omega_{\mathrm{X}}$ & $\begin{array}{l}\text { gemessene Winkelgeschwindigkeit um die Fahzeuglängsachse (Wank- } \\
\text { rate) }\end{array}$ & $\mathrm{rad} / \mathrm{s}$ \\
\hline$\omega_{\mathrm{Z}}$ & $\begin{array}{l}\text { gemessene Winkelgeschwindigkeit um die Fahrzeughochachse (Gier- } \\
\text { rate) }\end{array}$ & $\mathrm{rad} / \mathrm{s}$ \\
\hline$\dot{\omega}_{\mathrm{X}}$ & gemessene Wankwinkelbeschleunigung & $\mathrm{rad} / \mathrm{s}^{2}$ \\
\hline$\dot{\omega}_{\mathrm{Z}}$ & Gierbeschleunigung um die Fahrzeughochachse & $\mathrm{rad} / \mathrm{s}^{2}$ \\
\hline
\end{tabular}




\section{Indizes}

Indize Beschreibung

\begin{tabular}{ll}
\hline Corr & Correvit \\
$\mathrm{E}$ & im erdfesten Koordinatensystem \\
$\mathrm{f}$ & an der Vorderachse \\
$\mathrm{fl}$ & Vorderachse links \\
$\mathrm{fr}$ & Vorderachse rechts \\
$\mathrm{r}$ & an der Hinterachse \\
$\mathrm{rl}$ & Hinterachse links \\
$\mathrm{rr}$ & Hinterachse rechts \\
$\mathrm{S}$ & im Sensorkoordinatensystem \\
$\mathrm{V}$ & im Fahrzeugkoordinatensystem \\
$\mathrm{X}$ & in X-Richtung im jeweiligen Koordinatensystem \\
$\mathrm{Y}$ & in Y-Richtung im jeweiligen Koordinatensystem \\
$\mathrm{Z}$ & in Z-Richtung im jeweiligen Koordinatensystem
\end{tabular}




\section{Abkürzungen}

Kürzel vollständige Bezeichnung

\begin{tabular}{|c|c|}
\hline ABS & Antiblockiersystem \\
\hline ATP & Automotiv Testcenter Papenburg \\
\hline CG & Center of Gravity (Schwerpunkt) \\
\hline EG & Eigenlenkgradient \\
\hline EKF & erweitertes Kalman-Filter \\
\hline ESC & Electronic Stability Control \\
\hline ESM & Einspurmodell \\
\hline ESP & elektronisches Stabilisierungsprogramm \\
\hline FDM & Fahrdynamikmodell \\
\hline GPS & Global-Positioning-System \\
\hline HA & Hinterachse \\
\hline HK & Handlingkurs \\
\hline IMU & Inertial-Measurement-Unit \\
\hline $\mathrm{KIN}$ & kinematisches Modell \\
\hline $\mathrm{KF}$ & Kalman-Filter \\
\hline LS & Least-Square \\
\hline $\max$ & maximal \\
\hline $\min$ & minimal \\
\hline NLB & nichtlinearer Beobachter \\
\hline PT1 & Verzögerungsglied 1. Ordnung \\
\hline PT2 & Verzögerungsglied zweiter Ordnung \\
\hline PZB & Prüfzentrum Boxberg \\
\hline $\mathrm{RC}$ & Rollcenter (Wankpol) \\
\hline RLS & Recursive-Least-Square \\
\hline STM & Single Track Model \\
\hline rms & root mean square \\
\hline SG & Schwimmwinkelgradient \\
\hline SR & Sommerreifen \\
\hline $\mathrm{UKF}$ & Unscented Kalman-Filter \\
\hline WG & Wankwinkelgradient \\
\hline WI & Wankindex \\
\hline WR & Winterreifen \\
\hline VA & Vorderachse \\
\hline ZVF & Zustandsvariablenfilter \\
\hline ZSM & Zweispurmodell \\
\hline
\end{tabular}




\section{Kurzfassung}

Ziel dieser Arbeit war die Entwicklung einer Methode zur Schätzung der wichtigsten Bewegungsgrößen Schwerpunktgeschwindigkeit und Schwimmwinkel, um diese einer Fahrdynamikregelung zur Verfügung zu stellen. Dazu sollte lediglich die Sensorik der Electronic Stability Control (ESC) genutzt werden. Damit eine modellbasierte Steuerung oder ein Führungsmodell auf Veränderung des querdynamischen Fahrverhaltens reagieren kann, sollten zusätzlich die fahrdynamisch wichtigsten Parameter, wie die Schräglaufsteifigkeiten und der maximale Reibwert während des Fahrbetriebs geschätzt werden.

Fahrdynamische Modelle wurden auf Basis der Starrkörperbewegung im Raum entwickelt, die alle erforderlichen Situationen, wie die Fahrt in der Steilkurve, Fahrten im physikalischen Grenzbereich auf Hoch- und Niedrigreibwert genau genug modellieren. Dabei wurde ein optimaler Kompromiss aus Komplexität und Genauigkeit gefunden.

Die fahrzeug- und reifenabhängigen Parameter dieser nichtlinearen Schlupf-Kraftmodelle wurden durch Fahrversuche und einer neu entwickelten Referenzsensorik-Konfiguration bestehend aus 6D-IMU, GPS und Correvit-Sensor identifiziert. Dabei wurden erstmals auch Manöver mit gleichzeitigem Längs- und Querschlupf (Kurvenbremsungen) verwendet, um die Querkraftabschwächung in Abhängigkeit des Längsschlupfes zu parametrieren. Die Validierung mit einer Kurvenbremsung bei maximaler Querbeschleunigung zeigt die Leistungsfähigkeit des resultierenden Fahrdynamikmodells auf.

Die Schätzung der fahrdynamischen Zustände und Parameter mit ESC-Sensorik wurde auf Basis von erweiterten und Unscented Kalman-Filtern entwickelt. Die Prädiktion der Zustände erfolgte wie in der Luft- und Raumfahrt üblich mit einem kinematischen Modell, d.h. durch Integration der Längs- und Querbeschleunigungsensorsignale, sowie der Gierrate. Die Korrektur dieser instabilen Integration erfolgte durch die Geschwindigkeiten der Vorderräder und mit den (aus Sensorgrößen geschätzten) Längs- und Querkräften der Vorder- und Hinterachse. Durch Berücksichtigung des maximalen Reibwerts in den Achslängs- und Querkraftmodellen wird dieser bei genügend Schlupf beobachtbar.

Es konnte gezeigt und erstmals begründet werden, warum der hier zur Zustandsschätzung eingesetzte Unscented Kalman-Filter im fahrdynamischen Grenzbereich durch die Berücksichtigung der Nichtlinearität den Schwimmwinkel robuster als der erweiterte Kalman-Filter schätzt.

Die Schwimmwinkelschätzung wurde mittels 355 unterschiedlicher, vom Autor selbst durchgeführter Testfahrten auf Fahrdynamikflächen, Steilkurven, Handlingkursen und auf Schnee validiert. Der Algorithmus lieferte auf ca. 1000 Testfahrt-Kilometern in allen erdenklichen fahrdynamisch relevanten Situationen robuste Ergebnisse. Im Mittel betrug der maximale Schwimmwinkelfehler während einer Testfahrt 2,7 . Der entwickelte Schwimmwinkelschätzer kann daher einen entscheidenden Beitrag bei der Weiterentwicklung des ESC leisten, indem insbesondere 
kritische Situationen frühzeitig erkannt werden. Die Reibwertschätzung reagierte sehr schnell, sodass bereits bei ca. 80-85\% der maximalen Querbeschleunigung der maximale Reibwert richtig eingeschätzt wurde. Die Schräglaufsteifigkeiten konnten während einer Landstraßenfahrt mit mittleren Querbeschleunigungen robust geschätzt werden. Der Unterschied zwischen einer Winterund Sommerbereifung wurde deutlich.

Um das Zusammenspiel des entwickelten Schätzalgorithmus mit einer Fahrdynamikregelung zu demonstrieren, wurde eine Modellfolgesteuerung einer aktiven Vorder- und Hinterachslenkung zur Verbesserung der Gierdynamik in einer IPG-Carmaker-Simulation implementiert. Durch die Rückführung des geschätzten Schwimmwinkels und einem einfachen P-Regler konnte das Fahrzeug durch Bremseingriffe auch bei einem langsam anwachsenden Schwimmwinkeln frühzeitig stabilisiert werden, was durch eine Gierratenrückführung nicht möglich war. 